\title{
The Present and Future of Research by the International Biophilia Rehabilitation Academy
}

\author{
Toshiyuki Tanaka \\ Professor, Ph.D \\ Department of Applied Physics and Physico-Informatics, \\ Faculty of Science and Technology, Keio University
}

\begin{abstract}
The goal of Biophilia Rehabilitation Academy (BRA) is to clarify the relation between a brain function and a paralysis, and to perform the therapy of stroke patients using the knowledge of the previous researches. Before now, I have proposed that doctors diagnose the recovery level of paralysis by measuring brain function at rehabilitation as well as by rehabilitation scores, and give the adequate medical treatment to the patients. In the 12th International Biophilia Rehabilitation Conference at Keio university, the research group of BRA started with endeavor that our contribution reaches in one of the medical science. I show in this report about the previous, current and future researches by the international Biophilia Rehabilitation Academy. Although many researchers have made effective devices for rehabilitation in some countries, most of the devices are expensive and requires some infrastructures. Our investigative group has tried to make a system of rehabilitation with cheap and effective devices, which we call it "Takizawa method". The exercises in Takizawa method is motivative exercise, and the traditional method in rehabilitation is passive exercise. We think that Takizawa method will be a standard rehabilitation system in the future in many countries.
\end{abstract}

Keywords: Takizawa method, Rehabilitation system, motivative exercise

\section{Introduction}

In Japan the number of stroke patients have been increasing year by year, because of aging population. Since a traditional rehabilitation system cannot tackle the increasing stroke patients, many doctors and researchers have worked on the problems of system innovation. In some solutions for new rehabilitation, new devices for rehabilitation have been developed and new methods of therapy have been proposed in some organization such as hospital and university. However, most of the solutions require the expensive devices and infrastructures. Therefore, only wealthy persons can utilize the systems. We require new devices and system that many economically-challenge families can use in the world. In such a situation, Takizawa method ${ }^{1)}$ has appeared as rehabilitation system in Japan. In this method, one physical therapist (PT) can examine some patients with simple and cheap devices. Moreover, the patients can use the same devices at home. One dubious point is that Takizawa method is more or similarly effective than the traditional rehabilitation system. Our members have been checking the effectiveness of Takizawa method by PT score and some approach of engineering ${ }^{2)}$. The recent results of our measurements shows that Takizawa method is effective as future rehabilitation in the world. Ministry of Internal Affairs and Communications in Japan recognizes our efforts and adopts our proposal to the competitive funds SCOPE. We have made effort to sublimation of our contribution to science, and I pay a central role in keeping it up. I hope as the conference chair that this conference become a starting point for paper publication and a global corporation with many countries.

\section{Takizawa method}

In Takizawa method, some devices are improved so that a patient can privately buy and use it at home. Although some devices are so large for patients, it is supposed that those devices are use at the hospital. The private and portable devices "Pata" and "Koro" is shown in Fig. 1. One PT teaches how to use the devices to some patients. One PT can examine several patients simultaneously. In the system with Takizawa method, since even small hospital can hire PT, we think that it will result in leaping in employment of PT. In the recent study, many patients improved their body impaired function. It is hoped that many persons will be helped by Takizawa method, in the future aging society. 


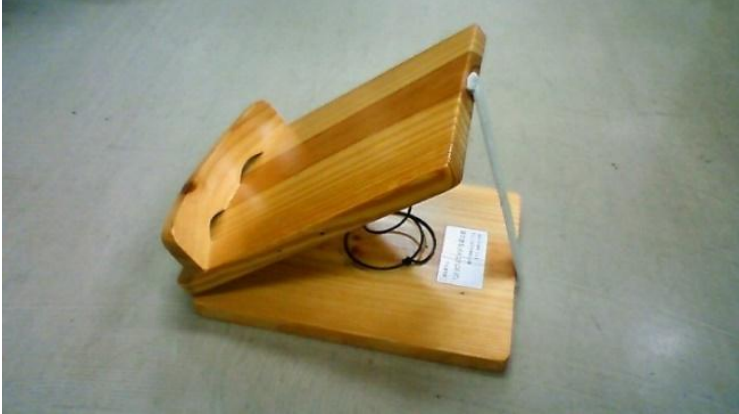

(a) A patients knocks over this devices, "Pata".

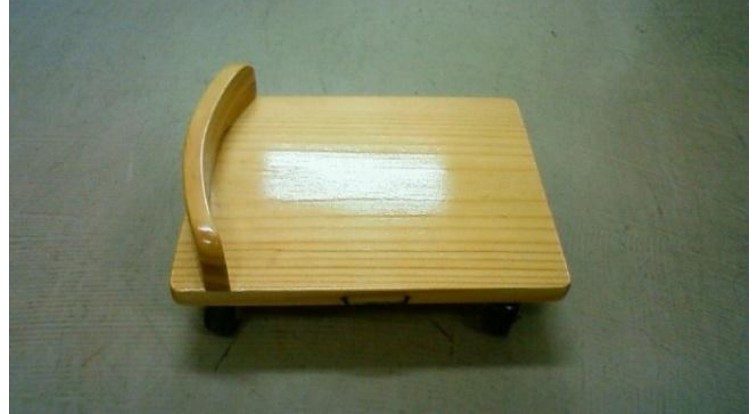

(b) A patient rolls over this device, "Koro".

Fig. 1 Examples of devices proposed in Takizawa method

\section{Evaluation for Takizawa method}

In order to check Takizawa method, we clarified the characteristics of the total functional independent measure (FIM) score and the relation between the number of improved items. Furthermore, we investigated the multi-dimensional relevance of 18 FIM items. Finally, we statistically verified the effect about 18 FIM evaluation items ${ }^{3)}$. Next, we performed a randomized controlled trial ${ }^{4)}$. Although exercise intervention for frail elderly persons was not so interested in, the exercise has drawn attention as the method for preventing and improving condition thereafter enforcement of the Long-Term-Care Insurance Law and the revision of the long-term care insurance system. In randomized controlled trial, 145 female patients were admitted to special nursing homes. They were stratified according to their care levels and randomly assigned to either the exercise therapy invention group or control group. In the results, the functional independence measure score did not improve in the exercise therapy invention group.

\section{Evaluation by engineering method}

Recently, we evaluate the Takizawa method by some engineering method. One method is based on mechanical system, and other is signal processing method. Since objective evaluation of rehabilitation is so difficult, although our research group also check the activation site in brain by functional near-infrared spectroscopy (fNIRS), we have not sufficiently obtained the results of objective evaluation.

\section{Conclusions}

Takizawa method is expected for the future rehabilitation system. Although it is known along the way that many stroke patients have been recovered by Takizawa method, the objective evaluation is not sufficiently obtained. Hereafter, we have no doubt that Takizawa method becomes widely used. We think that we compare Takizawa method with the traditional method, and must spread the objective advantage of Takizawa method without further delay.

\section{Acknowledgements}

This research and development work was supported by the MIC/SCOPE \#162303003.

\section{Reference}

1) Shigeo Takizawa, How to Invent the Autonomous Rehabilitation Method (Takizawa method), BIOPHILIA Vol. 2014 (2014) No. 1 p. 1-6

2) Shigeo Takizawa, Yoshiyasu TakeFuji, Tomoji Ishimaru, Rika Wada, Hajime Takada, Tetsuhiko Kimura, Construction of Community Biophilia Rehabilitation Network for the Disabled Elderly, BIOPHILIA Vol. 2015 (2015) No. 1 Memorial Edition for the Linking ISSN Registration p. 28-35

3) Kenji Ushizawa, Shigeo Takizawa, Hiroshi Nagasawa, Mitsuyo Makita, Tetsuhiko Kimura, kyoko Takizawa, Statistical Evaluation of Rehabilitation to the Disabled Elderly Based on the Takizawa Method, BIOPHILIA Vol. 2015 (2015) No. 1 Memorial Edition for the Linking ISSN Registration p. 19-27

4) Mitsuyo MAKITA, Hiroto NAKADAIRA, Masaharu YAMAMOTO, Randomized Controlled Trial to Evaluate Effectiveness of Exercise Therapy (Takizawa Program) for Frail Elderly, Environmental Health and Preventive Medicine Vol. 11 (2006) No. 5 P 221-227 